\begin{tabular}{|c|c|}
\hline Title & $\begin{array}{l}\text { Biological application of focus ion beam-scanning electron microscopy (FIB-SEM) to the imaging of cartilaginous } \\
\text { fibrils and osteoblastic cytoplasmic processes }\end{array}$ \\
\hline Author(s) & $\begin{array}{l}\text { Hasegawa, Tomoka; Endo, T akashi; T suchiya, Erika; Kudo, A i; Zhao, Shen; Moritani, Y asuhito; A be, Miki; } \\
\text { Y amamoto, T omomaya; Hongo, Hiromi; T suboi, Kanako; Y oshida, Taiji; Nagai, T omoya; Khadiza, Naznin; } \\
\text { Y okoyama, A yako; Luiz de Freitas, Paulo Henrique; Li, Minqi; A mizuka, Norio }\end{array}$ \\
\hline Citation & $\begin{array}{l}\text { Journal of Oral Biosciences, 59(1), 55-62 } \\
\text { https://doi.org/10.1016/.job.2016.11.004 }\end{array}$ \\
\hline Issue Date & 2017-02 \\
\hline Doc URL & http:/hdl.handle.net/2115/68253 \\
\hline Rights & (C) 2017. This manuscript version is made available under the CC-BY-NC-ND 4.0 license \\
\hline Rights(URL) & http://creativecommons.org/icenses/by-nc-nd/4.0/ \\
\hline Type & article (author version) \\
\hline File Information & J oral biosci59(1).pdf \\
\hline
\end{tabular}

Instructions for use 


\title{
Biological application of focus ion beam-scanning electron microscopy (FIB-SEM) to the imaging of cartilaginous fibrils and osteoblastic cytoplasmic processes
}

\author{
Tomoka Hasegawa $^{1 \S}$, Takashi Endo ${ }^{2}$, Erika Tsuchiya ${ }^{1,3}$, Ai Kudo ${ }^{1}$, Zhao Shen ${ }^{1,4}$, \\ Yasuhito Moritani ${ }^{4}$, Miki Abe ${ }^{1}$, Tomomaya Yamamoto ${ }^{1}$, Hiromi Hongo ${ }^{1}$, Kanako \\ Tsuboi $^{1,3}$, Taiji Yoshida ${ }^{1}$, Tomoya Nagai ${ }^{1,5}$, Naznin Khadiza ${ }^{1,6}$, Ayako Yokoyama ${ }^{1,7}$, \\ Paulo Henrique Luiz de Freitas ${ }^{8}$, Minqi Li $^{9}$ and Norio Amizuka ${ }^{1}$
}

${ }^{1}$ Department of Developmental Biology of Hard Tissue, Graduate School of Dental Medicine, Hokkaido University, Sapporo, Japan. ${ }^{2}$ Laboratory of Nano-Micro Material Analysis, Joint-use Facilities, Faculty of Engineering, Hokkaido University, Sapporo, Japan. Departments of ${ }^{3}$ Oral Diagnosis and Medicine, ${ }^{4}$ Oral and Maxillofacial Surgery, ${ }^{5}$ Oral Functional Prothodontics, ${ }^{6}$ Dentistry for Children and Disabled Person, ${ }^{7}$ Gerodontology, Graduate School of Dental Medicine, Hokkaido University, Sapporo, Japan. ${ }^{8}$ Department of Dentistry, Federal University of Sergipe at Lagarto, Brazil. ${ }^{9}$ Division of Basic Science of Stomatology, The School of Stomatology, Shandong University, Jinan, China.

\section{Running title: FIB-SEM observation on cartilage and bone}

\author{
${ }^{\S}$ Corresponding Author
}

Tomoka Hasegawa, DDS, Ph.D

Department of Developmental Biology of Hard Tissue, Graduate School of Dental Medicine, Hokkaido University

Kita 13, Nishi 7, Kita-Ku, Sapporo, Hokkaido, 060-8586, Japan

$$
\text { Tel/fax: +81-11-706-4226 }
$$

E-mail: hasegawa@den.hokudai.ac.jp 


\section{Abstract}

Objectives: The aim of this study is a biological application of focused ion beam-scanning electron microscopy (FIB-SEM) to demonstrate serial sectional images of skeletal tissues, here presenting the ultrastructure of 1) cartilaginous extracellular fibrils and 2) osteoblastic cytoplasmic processes.

Methods: Seven weeks-old female wild-type mice were fixed with half-Karnovsky solution and subsequent $\mathrm{OsO} 4$, and the tibiae were extracted for block staining prior to observation under transmission electron microscope (TEM) and FIB-SEM.

Results: TEM showed the fine fibrillar, but somewhat amorphous ultrastructure of the intercolumnar septa in the growth plate cartilage. Alternatively, FIB-SEM revealed bundles of stout fibrils at regular intervals paralleling the septa's longitudinal axis, as well as vesicular structures embedded in the cartilaginous matrix of the proliferative zone. In the primary trabeculae, both TEM and FIB-SEM showed several osteoblastic cytoplasmic processes on the osteoid, with numbers higher than those seen in the bone matrix. FIB-SEM revealed the agglomeration of cytoplasmic processes beneath the osteoblasts, which formed a tubular continuum extending from those cells. Based on these findings, we postulated that osteoblasts not only extend their cytoplasmic processes through to the bone matrix, but also stack these cell processes on the osteoid of the primary trabeculae.

Conclusion: Taken together, it is likely that FIB-SEM imaging strategy on serial sections may successfully deliver new insights on the ultrastructure of cartilage and bone tissues.

Key words: FIB-SEM, chondrocyte, osteoblast, ultrastructure, cartilage matrix 


\section{Introduction}

Focused ion beam-scanning electron microscopy (FIB-SEM), also referred to as ion abrasion scanning electron microscopy (1-3) may be one of the most valuable techniques to assess tissue morphology at the ultrastructural level, especially for cartilage and bone research. Usually, FIB-SEM observations employs epoxy resin-embedded specimens that are ground by a gallium focused ion beam (4). Then, the resultant surface images is detected by scanning electron microscopy (SEM) backscattering, a process known as "slice-and-view" $(5,6)$. While targeting specific regions of a given tissue sample can be a challenging task with transmission electron microscopy (TEM), FIB-SEM may overcome such limitation by employing faster visualization of serial ultra-thin section. However, few studies have explored the use of FIB-SEM for the ultrastructural assessment of bone and cartilage (7).

Histologically, the developing growth plate cartilage comprises three major zones: the reserve or resting zone, the proliferative zone and the hypertrophic zone (8). Chondrocytes in the proliferative zone proliferate and synthesize cartilaginous matrix simultaneously (9). Cells from that zone become flattened proliferative chondrocytes, align themselves in columns and, after that, enter the hypertrophic phenotype marked by a translucent and enlarged cell body $(8,10)$. The cartilaginous extracellular matrix in these zones can be divided into two regions: the intercolumnar septa, which parallel the longitudinal cell columns, and the transverse partitions within those columns (11). However, if external mechanical loading is applied to the growth plate cartilage, the ultrastructural relation between chondrocytes and extracellular fibrils can be dramatically affected and disrupt the chondrocytic columns (12). Therefore, it is important to determine the normal ultrastructure of extracellular cartilaginous fibrils through FIB-SEM observations so that any existing disorders can be readily identified.

As a consequence of normal endochondral ossification $(8,13)$, osteoblasts extend their cytoplasmic processes into the bone matrix of the primary trabeculae $(14,15)$. 
Hasegawa et al.

Since bone formation rate is extremely high in primary trabeculae (16-18), one may wonder whether an osteoblast can produce new cytoplasmic processes at the same rate that it forms new bone. Alternatively, is it possible that cytoplasmic processes are synthesized in advance and "stocked” beneath the osteoblasts until needed?

In this study, we present the ultrastructure of 1) cartilaginous extracellular fibrils and 2) osteoblastic cytoplasmic processes by means of FIB-SEM. In addition, we present a modification of the block staining procedures reported by Thomas et al (19) in an effort to obtain adequate contrast in backscattered SEM images. 


\section{Materials \& Methods}

\subsection{Tissue preparation for TEM and FIB-SEM}

All animal experiments were conducted under the Hokkaido University Guidelines for Animal Experimentation (approval No.15-0041). Seven weeks-old female wild-type mice (Jcl: ICR, CLEA Japan) were used in this study ( $\mathrm{n}=6)$.

Mice were anesthetized with an intraperitoneal injection of sodium pentobarbital and perfused with half-Karnovsky solution (2\% paraformaldehyde and 2.5\% glutaraldehyde diluted in a 0.067M cacodylate buffer, $\mathrm{pH} 7.4$ ) through their left heart ventricle. After perfusion, all tibiae were stripped of soft tissues and immediately immersed in the same fixative for $48 \mathrm{~h}$ at $4{ }^{\circ} \mathrm{C}$. After decalcification with a solution of 4.13\% EDTA for two months, the specimens were cut into small pieces of $2 \mathrm{~mm} \mathrm{x} 1 \mathrm{~mm}$ $\mathrm{x} 1 \mathrm{~mm}$ that included the metaphyseal growth plate. Specimens were subjected to block staining as described below, and were dehydrated with ascending concentrations of acetone before embedding in epoxy resin (Taab, Berkshire, UK) (20). Semi-thin sections were obtained from the surfaces of the embedded specimens and observed under a light microscope after staining with toluidine blue (Merck, Darmstadt, Germany) to determine the area to be analyzed through FIB-SEM (JIB-4600F, JEOL Ltd., Tokyo, Japan). Ultra-thin sections were obtained with a microtome (Sorvall MT-5000, Du Pont, DD) for TEM observation at 80 kV (Hitachi H-7100 Hitachi Co. Ltd, Tokyo, Japan).

\subsection{Block staining for FIB-SEM}

The method proposed by Thomas et al (19) for block staining prior to FIB-SEM imaging was modified to optimize contrast in backscattered SEM images of bone and cartilage. Contrast optimization is necessary because the decalcification process used for mineralized tissues is completely different from that used in the preparation of specimens from soft, non-mineralized tissues such as nerves. 
Specimens (2mm x 1mm x 1mm) were embedded in 6\% agarose gel and sliced into $300 \mu$ m-thick sections. These sections were then incubated in a solution of $2 \%$ OsO4 and $3 \%$ potassium ferrocyanide in a $0.067 \mathrm{M}$ cacodylate buffer with $4 \mathrm{mM}$ calcium chloride for $5 \mathrm{~h}$ at $4{ }^{\circ} \mathrm{C}$, which is considerably longer than suggested by Thomas et al (19). Then, specimens were washed with distilled water in a rotator for 3 days, with distilled water being changed 3 times a day. After washing, specimens were immersed in $1 \%$ thiocarbohydrazide for $4 \mathrm{~h}$ at room temperature (RT), treated with $2 \% \mathrm{OsO}_{4}$ for 3 $\mathrm{h}$ at RT, and placed in saturated uranyl acetate overnight at $4{ }^{\circ} \mathrm{C}$. After washing with distilled water as described above, the specimens were stained with en bloc Walton's lead aspartate solution ( $0.066 \mathrm{~g}$ of lead nitrate in $10 \mathrm{ml}$ of a $0.03 \mathrm{M}$ aspartic acid solution). Specimens were washed in distilled water for 3 days once again before dehydration with ascending acetone solutions.

\subsection{FIB-SEM observation}

Tibial specimens were mounted in a special holder and sputter-coated with platinum to obtain conductive layers on the cylinder's lateral and basal walls. Serial images of the block's surface were acquired through repeated cycles of sample surface milling using a $30 \mathrm{kV}$ focused gallium ion beam at $3 \mathrm{nA}$. The dimensions of the region of interest for FIB-SEM observation were $30 \mu \mathrm{m} \quad$ x $20 \mu \mathrm{m} \quad$ x $17.5 \mu \mathrm{m}$ (ROI, See Fig.1). Approximately 350 consecutive slices with 50 nm-thickness were obtained. 


\section{Results}

\subsection{Chondrocytes and surrounding cartilage fibrils}

The ultrastructure of cartilaginous fibrils surrounding chondrocytes in the proliferative and hypertrophic zones assessed by TEM and FIB-SEM were compared, as seen in Fig.

2. TEM imaging showed the transverse partitions of the chondrocytic columns to be composed of non-fibrillar amorphous components, while FIB-SEM imaging showed randomly oriented fibrillar structures in a corresponding region (Figs. 2C, D). In contrast, TEM showed the dense assembly of fine fibrillar (but somewhat amorphous) structures in the longitudinal intercolumnar septa, whereas FIB-SEM unveiled stout fibrils running in parallel with some space between them (Figs. 2E, F). Thus, TEM imaging revealed the structure of cartilaginous fibrils, whereas FIB-SEM images tended to demonstrate evident fibrillar structure with high contrast in cartilage.

At intervals of $2 \mu \mathrm{m}$, FIB-SEM imaging revealed the bundles of cartilaginous fibrils running parallel to the longitudinal axis of the chondrocytic columns, as well as vesicular materials deposited in the intercolumnar cartilaginous septa (Fig. 3). Observed at a higher magnification and with intervals of $150 \mathrm{~nm}$, FIB-SEM images displayed focal contact points between the short cytoplasmic processes of chondrocytes and the cartilaginous fibrils that ran longitudinally in the septa (Figs. 4A-L). In addition, the serial sections clearly showed that the included vesicular materials seen in Fig. 3 were spherical structures that were not connected to the surrounding chondrocytes (See arrows in Figs. 4A-E). The transverse partitions displayed focal contact points between the short chondrocytic cytoplasmic and the cartilaginous fibrils. The spherical structures embedded in the cartilaginous matrix were not as numerous as in the longitudinal intercolumnar septa (Figs. 4M-X). 
Hasegawa et al.

\subsection{Osteoblastic cytoplasmic processes in the primary trabeculae}

TEM and FIB-SEM images showed an agglomeration of cytoplasmic processes beneath the osteoblasts; consistently, the number of cytoplasmic processes seemed lower in the bone matrix than in the osteoid (Compare asterisks and arrows in Figs. 5C, D). However, it is possible that tubular structures (i.e., cytoplasmic processes) and spherical structures (i.e., vesicles) appear as round shapes in cross sections. As shown in Fig. 6, FIB-SEM imaging of $150 \mathrm{~nm}$-thick serial sections demonstrated the tubular nature of the cytoplasmic processes, since a single process is seen as a round structure in the serial images (arrowheads in Fig. 6). 


\section{Discussion}

To our knowledge, this is the first study employing the FIB-SEM imaging strategy to assess the cartilaginous fibrils and the osteoblastic cytoplasmic processes in the primary trabeculae. One important methodological choice was the modification of the block staining protocol reported by Thomas et al (19), which led to improved contrast of backscattered SEM images and, consequently, better ultrastructural image production. Bone and cartilage are abundant with collagen fibrils (8, 20, 21), which facilitate the deposition of uranyl acetate and lead aspartate. Therefore, it was necessary to extend block staining for a longer period, as well as to repeat the washing procedures to obtain FIB-SEM images of enough resolution so that proper comparison with TEM images was possible.

Still, there were subtle differences between TEM and FIB-SEM imaging. While the ultrastructure of plasma membranes imaged with the two techniques were similar, the cartilaginous matrix seemed composed of non-fibrillar amorphous components in TEM images but FIB-SEM pictures showed obviously fibrillar structures (Fig. 2). Thus, TEM imaging shows amorphous materials among the cartilaginous fibrils, while backscattering preferably reveals fibrillary structures. This discrepancy may be due to the image-detecting methods, - backscatter electron and transmitted electron - between FIB-SEM and TEM. Therefore, each technique may have its own advantage for envision the ultrastructures. When incident electrons illuminate specimen, a part of electrons is reflected backward and emitted from the specimen surface. The intensity of emitted electrons - backscattered electron is stronger as the atomic number of the constituent atoms is larger, and also the image of backscattering can show more intense contrast than transmitted electron images (22). Therefore, we postulate that TEM images show more detailed structures including non-fibrillar amorphous components, while FIB-SEM can provide images with highly-strengthened contrast of fibrillar structure in cartilaginous matrix. 
The main advantage of FIB-SEM imaging is permitting faster visualization of serial ultra-thin sections, which optimizes the adjustments needed for identification of the target structures $(23,24)$. As a result, we confirmed that chondrocytes in the proliferative and the hypertrophic zones extend their short cytoplasmic processes to the cartilaginous fibrils that parallel the longitudinal axis of the growth plate, and that vesicular structures are present in the intercolumnar septa - even at the proliferative zone (Figs. 3 and 4). These extracellular vesicles may be "matrix vesicles" secreted by proliferative chondrocytes, but it is well-known that mineralization takes place from the upper layer of the hypertrophic zone of the growth plate cartilage $(8,13,21)$. To identify the matrix vesicle, it is necessary to verify the presence of a variety of enzymes and membrane transporters involved in mineralization. Possibly, proliferative chondrocytes may secret matrix vesicles incompletely-equipped with theses enzymes and membrane transporters. In any case, our finding using FIB-SEM may be a trigger for further examination of matrix vesicles in cartilage.

As shown in Figs. 5 and 6, we could hypothesize that osteoblasts seem to be able to "stock" their cytoplasmic processes on the osteoid in the primary trabeculae, which is marked by a high bone formation rate $(17,18)$. We have previously demonstrated the distribution of osteocytes and their cytoplasmic processes were irregularly-arranged in the immature primary trabeculae, though they were regular in the mature cortical bone (14-16). It may be reasonable that osteoblasts' cytoplasmic processes also irregularly-oriented and accumulated in the osteoid. However, the geometrical nature of these structures must be confirmed through serial sections rather than observed on a single section. In fact, FIB-SEM has been recently employed to assess the three-dimensional aspects of several tissues using a specific imaging software (3, 25-27), and also in dental histology, where it revealed the connections among dentinal tubules (28). One limitation of this study is that only two-dimensional images were used; that being said, we are currently refining one three-dimensional FIB-SEM reconstruction protocol that will be presented to the bone research community in a near future. 


\section{Conclusion}

In this study, we have used a FIB-SEM imaging strategy to successfully demonstrate richer spatial information concerning the structure of extracellular cartilaginous fibrils and of osteoblastic cytoplasmic processes in metaphyseal primary trabeculae. Taken together, it is likely that the application of FIB-SEM imaging to the study of mineralized tissues is feasible and promising.

\section{Ethical approval}

The animal protocols used in this study were approved by the Hokkaido University Guidelines for Animal Experimentation (approval No.15-0041).

\section{Conflict of interest}

No potential conflicts of interest exist.

\section{Acknowledgements}

This work was conducted at "Joint-use Facilities: Laboratory of Nano-Micro Material Analysis”, Hokkaido University, supported by "Nanotechnology Platform” Program of the Ministry of Education, Culture, Sports, Science and Technology (MEXT), Japan. 


\section{References}

1. Drobne D, Milani M, Leser V, Tatti F, Zrimec A, Znidarsic N, Kostanjsek R, Strus J. Imaging of intracellular spherical lamellar structures and tissue gross morphology by a focused ion beam/scanning electron microscope (FIB/SEM). Ultramicroscopy 2008;108:663-70.

2. Heymann JA, Shi D, Kim S, Bliss D, Milne JL, Subramaniam S. 3D imaging of mammalian cells with ion-abrasion scanning electron microscopy. J Struct Biol 2009;166:1-7.

3. Knott G, Marchman H, Wall D, Lich B. Serial section scanning electron microscopy of adult brain tissue using focused ion beam milling. J Neurosci 2008; 28:2959-64.

4. Kizilyaprak C, Longo G, Daraspe J, Humbel BM. Investigation of resins suitable for the preparation of biological sample for 3-D electron microscopy. J Struct Biol 2015;189:135-46.

5. Wilson JR, Kobsiriphat W, Mendoza R, Chen HY, Hiller JM, Miller DJ, Thornton K, Voorhees PW, Adler SB, Barnett SA. Three-dimensional reconstruction of a solid-oxide fuel-cell anode. Nat Mater 2006;5:541-4.

6. Earl JS, Leary RK, Perrin JS, Brydson R, Harrington JP, Markowitz K, Milne SJ. Characterization of dentine structure in three dimensions using FIB-SEM. J Microsc 2010;240:1-5.

7. Hasegawa T, Yamamoto T, Haraguchi M, Amizuka N. Three-dimensional reconstruction of osteocytic lacunar-canalicular system in murine bone by using FIB-SEM. J Physiol Sci 2015;65(supp1): S185.

8. Amizuka N, Hasegawa T, Oda K, Luiz de Freitas PH, Hoshi K, Li M, Ozawa H. Histology of epiphyseal cartilage calcification and endochondral ossification. Front Biosci 2012;4: 2085-100.

9. Poole AR. The growth plate: cellular physiology, cartilage assembly and mineralization. In: Cartilage: Molecular Aspects. Ed. Hall BK \& Newman A, CRC Press, Boca Raton; 1991.

10. Amizuka N, Henderson JE, Hoshi K, Warshawsky H, Ozawa H, Goltzman D, Karaplis AC. Programmed cell death of chondrocytes and aberrant chondrogenesis in mice homozygous for parathyroid hormone-related peptide gene deletion. Endocrinology 1996 ;137:5055-67. 
11. Amizuka N, Warshawsky H, Henderson JE, Goltzman D, Karaplis AC. Parathyroid hormone-related peptide-depleted mice show abnormal epiphyseal cartilage development and altered endochondral bone formation. J Cell Biol 1994;126:1611-23.

12. de Freitas PH, Kojima T, Ubaidus S, Li M, Shang G, Takagi R, Maeda T, Oda K, Ozawa H, Amizuka N. Histological assessments on the abnormalities of mouse epiphyseal chondrocytes with short term centrifugal loading. Biomed Res 2007;28:191-203.

13. Ozawa H. Ultrastructural Concepts on Biological Calcification; Focused on Matrix Vesicles. J Oral Bioscience 1985;27:751-74.

14. Hongo H, Hasegawa T, Sasaki M, Suzuki R, Yamada T, Shimoji S, Yamamoto T, Amizuka N. Bone-Orchestrating Cells, Osteocytes. Hokkaido Journal of Dental Science 2012;32:93-103.

15. Sasaki M, Hongo H, Hasegawa T, Suzuki R, Liu Z, Freitas PHL, Yamada T, Oda K, Yamamoto T, Li M, Totsuka Y, Amizuka N. Morphological aspects of the biological function of the osteocytic lacunar canalicular system and of osteocyte-derived factors. Oral Science International 2012; 9:1-8.

16. Ubaidus S, Li M, Sultana S, de Freitas PH, Oda K, Maeda T, Takagi R, Amizuka N. FGF23 is mainly synthesized by osteocytes in the regularly distributed osteocytic lacunar canalicular system established after physiological bone remodeling. J Electron Microsc 2009;58:381-92.

17. Yamamoto T, Hasegawa T, Sasaki M, Hongo H, Tsuboi K, Shimizu T, Ota M, Haraguchi M, Takahata M, Oda K, Luiz de Freitas PH, Takakura A, Takao-Kawabata R, Isogai Y, Amizuka N. Frequency of Teriparatide Administration Affects the Histological Pattern of Bone Formation in Young Adult Male Mice. Endocrinology 2016;157:2604-20.

18. Takakura A, Takao-Kawabata R, Isogai Y, Kajiwara M, Murayama H, Ejiri S, Ishizuya T. Differences in vertebral, tibial, and iliac cancellous bone metabolism in ovariectomized rats. J Bone Miner Metab 2016;34:291-302.

19. Thomas JD, Eric AB, Andrea T, Mark HE. NCMIR METHODS FOR 3D EM: A new protocol for preparation of biological specomens for serial block face scanning electron microscopy. NCMIR 2010 Available at: https://www.ncmir.ucsd.edu/sbem-protocol/ [Accessed 29 Sep 2016].

20. Hasegawa T, Li M, Hara K, Sasaki M, Tabata C, de Freitas PH, Hongo H, Suzuki R, Kobayashi M, Inoue K, Yamamoto T, Oohata N, Oda K, Akiyama Y, Amizuka N. Morphological assessment of bone mineralization in tibial metaphyses of ascorbic acid-deficient ODS rats. Biomed Res 2011;32:259-69. 
21. Hasegawa T, Yamamoto T, Tsuchiya E, Hongo H, Tsuboi K, Kudo A, Abe M, Yoshida T, Nagai T, Khadiza N, Yokoyama A, Oda K, Ozawa H, Freitas de PHL, Li M, Amizuka N. Ultrastructural and biochemical aspects of matrix vesicle-mediated mineralization. Japanese Dental Science Review 2016; Epub ahead of print.

22. Goldstein JI, Newbury DE, Echlin P, Joy DC, Roming AD, Lyman CE, Fiori C, Lifshin E. Electron Optics. In: Scanning Electron Microscopy and X-Ray Microanalysis. Ed. Goldstein JI \& Newbury DE, Plunum Press, New York; 1992. p.21-25.

23. Drobne D, Milani M, Ballerini M, Zrimec A, Zrimec MB, Tatti F, Draslar K. Focused ion beam for microscopy and in situ sample preparation: application on a crustacean digestive system. J Biomed Opt 2004;9:1238-43.

24. Drobne D, Milani M, Zrimec A, Zrimec MB, Tatti F, Draslar K. Focused ion beam/scanning electron microscopy studies of Porcellio scaber (Isopoda, Crustacea) digestive gland epithelium cells. Scanning 2005;27:30-4.

25. De Winter DA, Schneijdenberg CT, Lebbink MN, Lich B, Verkleij AJ, Drury MR, Humbel BM. Tomography of insulating biological and geological materials using focused ion beam (FIB) sectioning and low-kV BSE imaging. J Microsc 2009;233:372-83.

26. Heymann JA, Hayles M, Gestmann I, Giannuzzi LA, Lich B, Subramaniam S. Site-specific 3D imaging of cells and tissues with a dual beam microscope. J Struct Biol 2006;155:63-73.

27. Ohta K, Sadayama S, Togo A, Higashi R, Tanoue R, Nakamura K. Beam deceleration for block-face scanning electron microscopy of embedded biological tissue. Micron. 2012;43:612-20.

28. Earl JS, Leary RK, Perrin JS, Brydson R, Harrington JP, Markowitz K, Milne SJ. Characterization of dentine structure in three dimensions using FIB-SEM. J Microsc 2010;240:1-5. 


\section{Figure Legends}

\section{Fig. 1}

\section{Region of interest (ROI) for FIB-SEM observation}

Schematic design of the FIB-SEM principle (A). The ROI is $30 \mu \mathrm{m} \times 20 \mu \mathrm{m} \times 17.5 \mu \mathrm{m}$ as indicated in the boxed area $b$. The boxed area $a$ is a pre-milled region used to observe the block surface and release the resin piece. The specimen is derived from the tibial metaphysis and includes the growth plate (B). Panel B shows the naked-eye view of the superficial layer sputter-coated with platinum. Ch: chondrocyte

\section{Fig. 2}

Ultrastructural images of cartilaginous fibrils observed by $\operatorname{TEM}(A, C, E)$ and $\operatorname{FIB-SEM}(B, D, F)$

Images of chondrocytes and the surrounding matrix at the transition between the proliferative and hypertrophic zones as imaged by TEM (A) and FIB-SEM (B). TEM images show non-fibrillar amorphous components in the transverse partitions $(C)$, while FIB-SEM shows randomly oriented fibrillar structures (D). Vesicular structures (arrows in panel F) can be seen tangled in the intercolumnar region when imaged through FIB-SEM. Note the stout, evident fibrillar structures. In contrast, TEM images show fine fibrillar structures with amorphous materials (E). Ch: chondrocyte Bars, A, B: $5 \mu \mathrm{m}$, C-F: $1 \mu \mathrm{m}$

\section{Fig. 3}

\section{Serial FIB-SEM imaging of chondrocytes and the surrounding extracellular fibrils}

Note the cartilaginous fibrils of the intercolumnar region paralleling the longitudinal axis of the cartilage (asterisks) as depicted by FIB-SEM imaging of $2 \mu \mathrm{m}$-thick serial sections. Note extracellular vesicular deposition as indicated by arrows.

Bars, $10 \mu \mathrm{m}$ 


\section{Fig. 4}

Serial FIB-SEM imaging of the intercolumnar region (A-L) and the transverse septum (M-X)

In $150 \mathrm{~nm}$-thick serial sections of the intercolumnar region, FIB-SEM images show focal contacts between the short chondrocytic cytoplasmic processes and the cartilaginous fibrils (See arrowheads in panel D, F, H). Note the extracellular vesicular structure (arrows in panel A-E) in panel A, which gradually becomes spherical and finally disappear in panel E. Consistently, the transverse partition reveals focal contact between the cartilage fibrils and the short chondrocytic processes (arrowheads, panels $\mathrm{S}$ and $\mathrm{T})$.

Bars, $2 \mu \mathrm{m}$

\section{Fig. 5}

Ultrastructural images of osteoblasts and their cytoplasmic processes obtained by $\operatorname{TEM}(A, C)$ and FIB-SEM $(B, D)$

TEM and FIB-SEM images demonstrate several vesicle-like structures on the osteoid (double arrows in A, B). At higher magnification, many stacked cytoplasmic processes are visible beneath the osteoblasts with both imaging techniques (asterisks in panels C and D). The number of cytoplasmic processes is markedly reduced in the bone matrix as compared to the osteoid (See arrows in panels C and D). ob: osteoblast, BM: bone matrix

Bars, A, B: $5 \mu \mathrm{m}, \mathrm{C}, \mathrm{D}: 2 \mu \mathrm{m}$

\section{Fig. 6}

\section{Serial FIB-SEM imaging of osteoblastic cytoplasmic processes}

The 150 nm-thick serial sections demonstrate the tubular nature of cytoplasmic processes (See arrows and arrowheads in all panels). Arrows indicate the tubular structure budding off the osteoblastic cell membrane and its extension from the budding. Arrowheads represent tubular structures in the two-dimensional plane. 
Hasegawa et al.

Bars, $2 \mu \mathrm{m}$ 

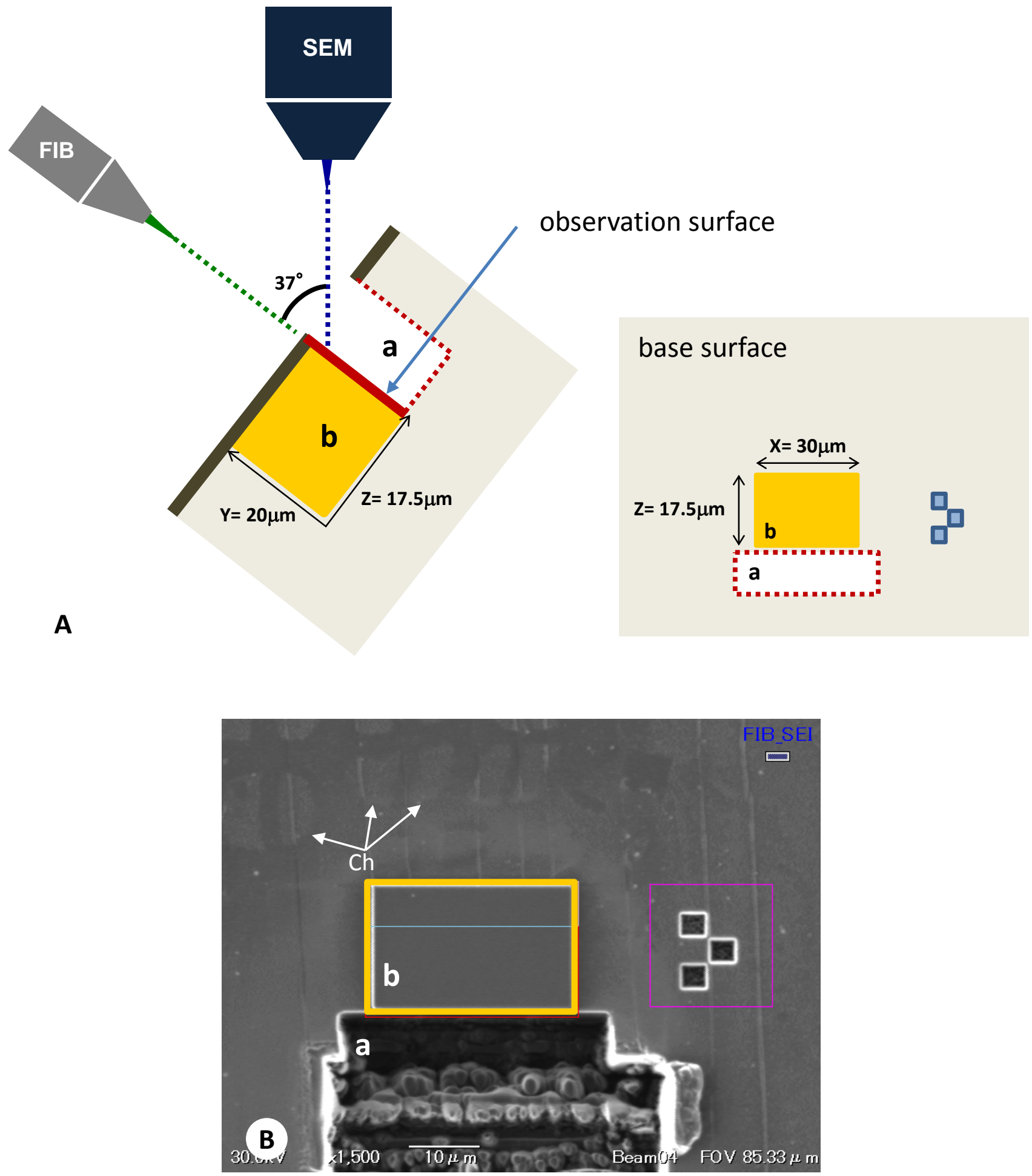

Figure 1 


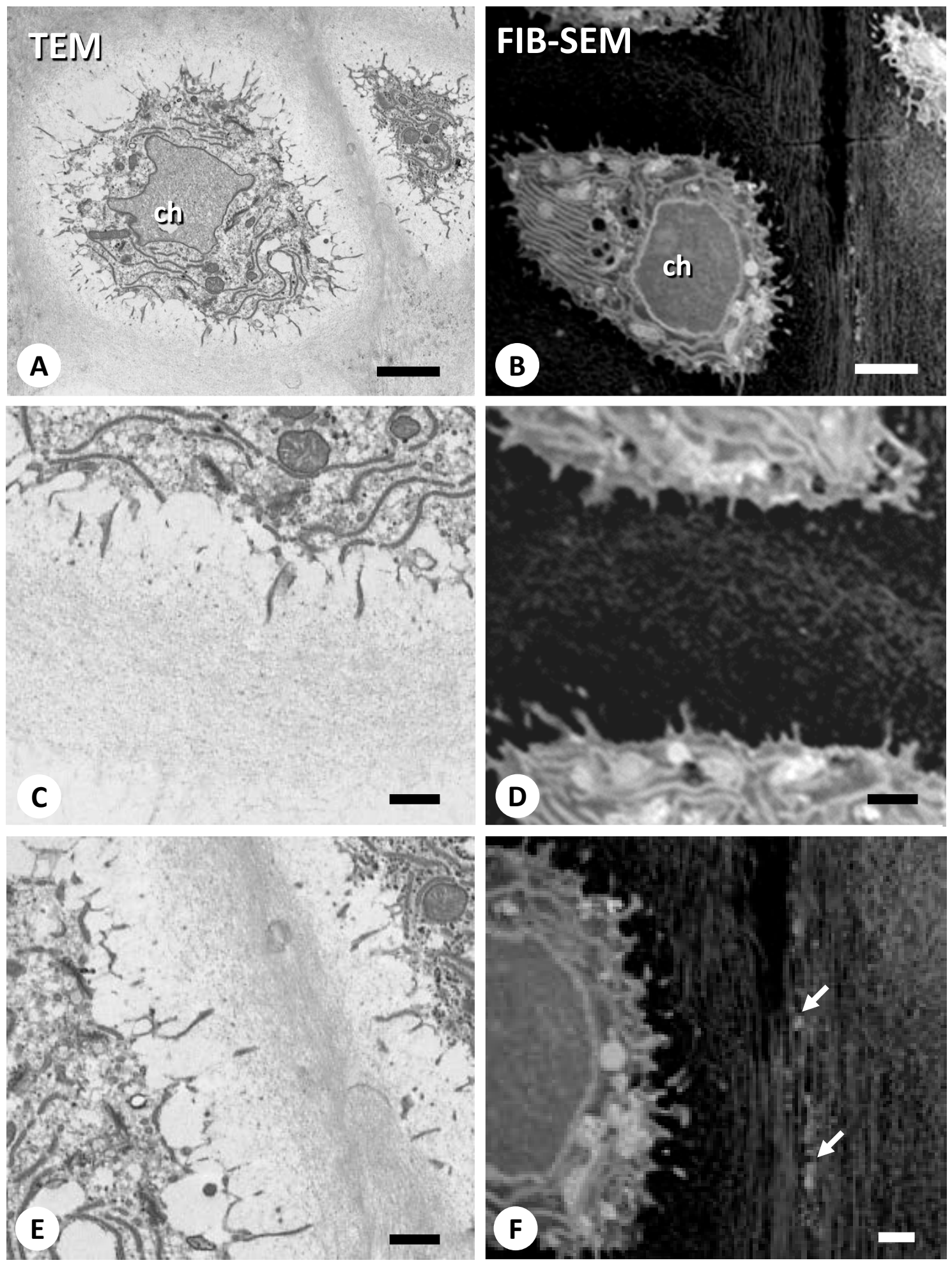

Figure 2 


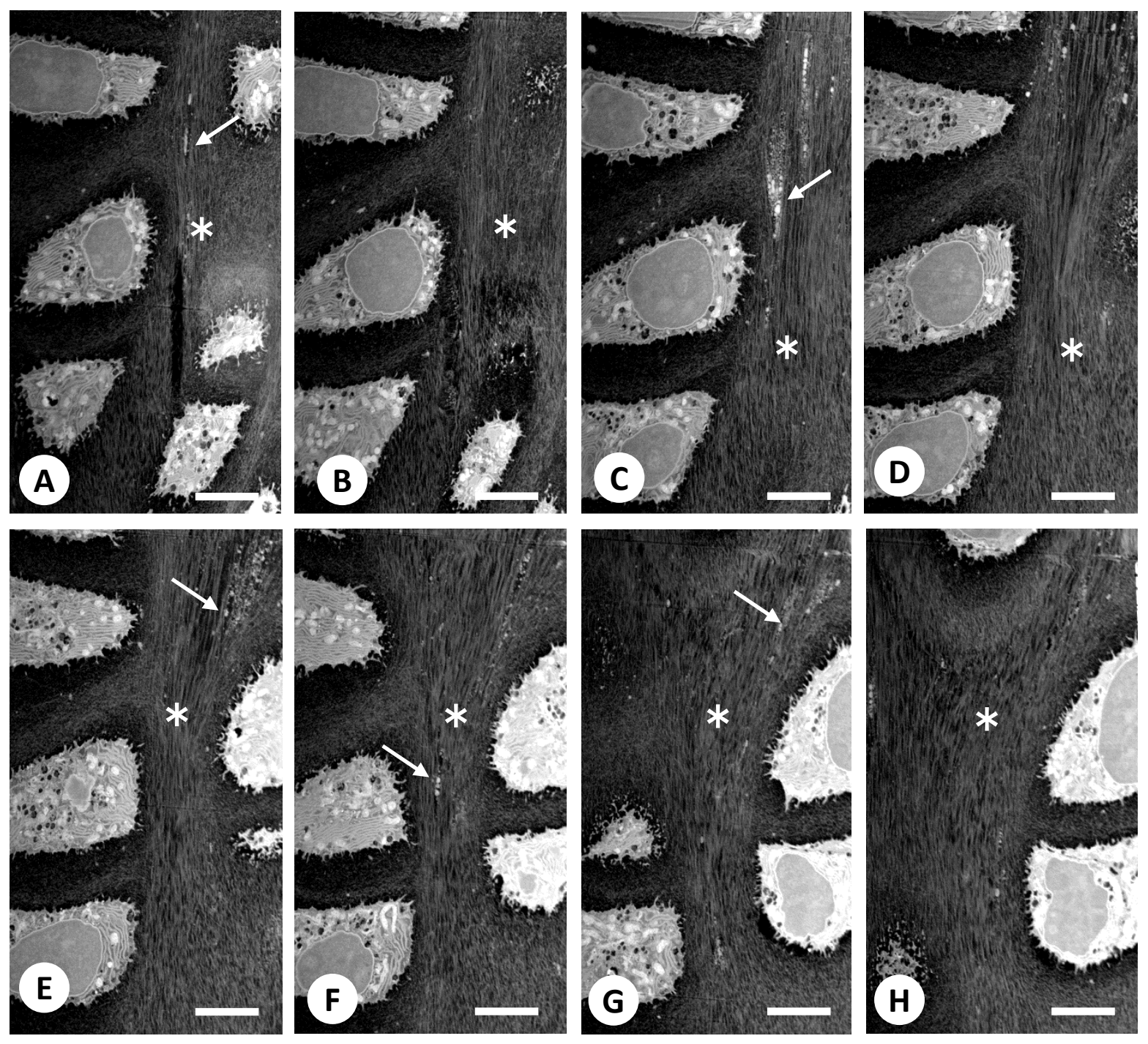

Figure 3 

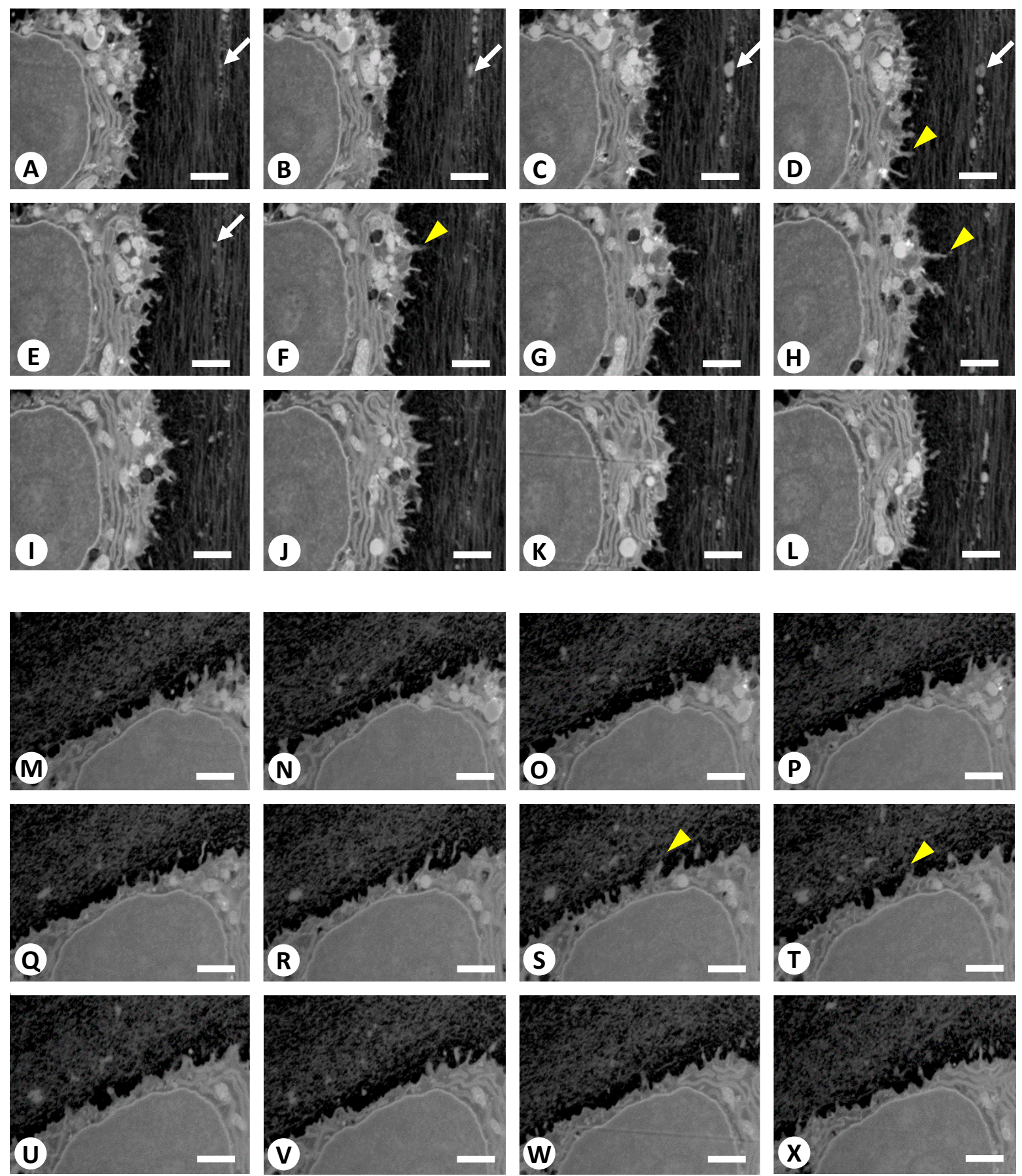

Figure 4 

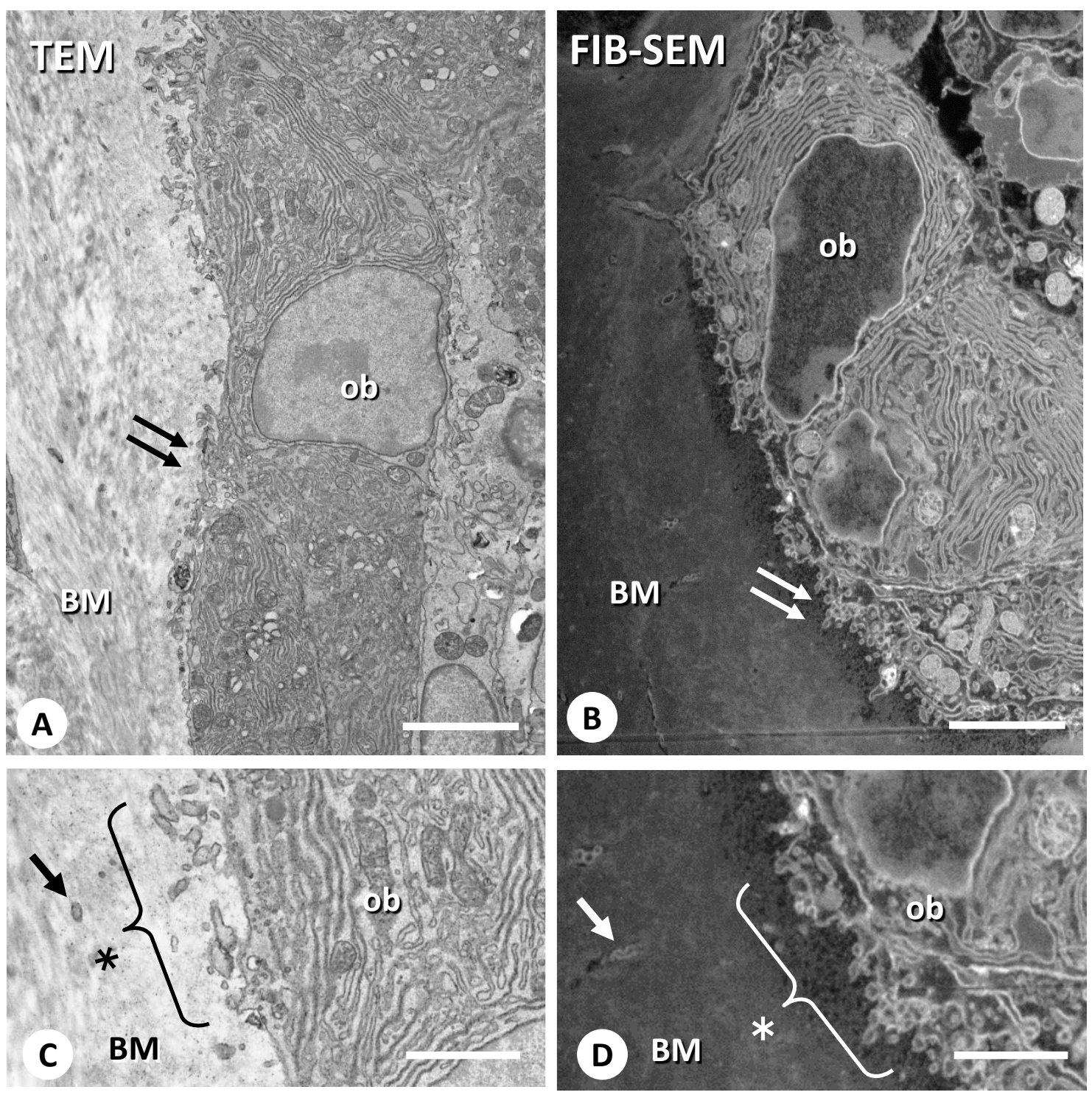

Figure 5 

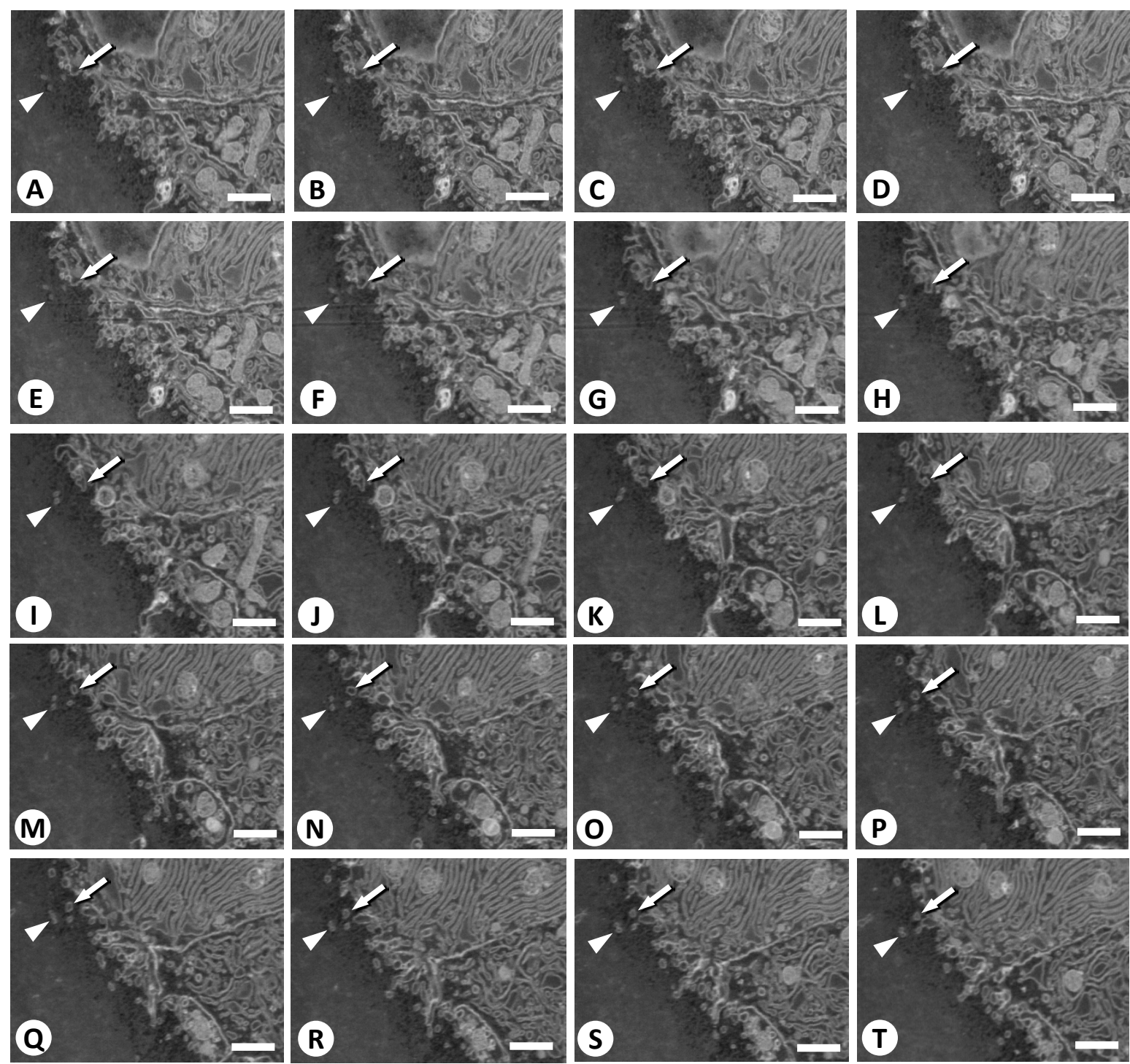

Figure 6 